She had 150 grs. hæmol and 120 grs. hæmogallol in a fortnight without the slightest benefit, while reduced iron improved her condition at once. GASE XIX.-Housemaid, aged 18, ill for some months. Date. Medicines.

May $2 . . . .$. Hæmol, 10 grs. thrice daily......3,700,000 ...... Hæmoglobin. Per Cent.
40

" 18...... Ferrum redactum, 2 grs.

Jüne $13 . . . . .$. thrice daily $\ldots . . .3,800,000 \ldots . . . .$. ......4,930,000 ..... .......4,500,000 . 9.......

44
34
72
80
89

In this case $480 \mathrm{grs}$. hæmol were taken in sixteen days, but the beneflt was very trifling.

One of the great problems in the study of chlorosis is to determine why the patient should not be able to renew the iron of the blood from the abundant iron of the food. Cases of acute hæmorrhage are generally able to do so, a few cases of chlorosis do so, but the great bulk of the latter only recover when inorganic iron is given. I have already stated my opinion that (for some as yet unknown reason) inorganic iron is much more helpful towards recovery than organic iron, although in health the latter serves perfectly for the manufacture of hæmoglobin, and is the only form in which iron is ordinarily taken by mankind.

Another point of great interest is to determine the reason why inorganic iron administered to the healthy in no way affects the number of red blood corpuscles or the amount of hæmoglobin. We have absolutely no knowledge as to how the use of iron by the organism is regulated, but it seems impossible to increase the number of corpuscles or the amount of hæmoglobin above a certain fixed normal. These and other matters must be left for future research.

In conclusion, I wish to touch on one other matter. It has been often stated that in chlorotics the amount of hæmoglobin is small and that it is impossible to raise it above a certain point, which is lower than the normal average; and it is pointed out that cases of chlorosis after treatment have seldom more than $\mathbf{7 0 . 8 0}$ per cent. hæmoglobin (Gowers's hæmoglobinometer). The following explanation of this has been given, and seems to me the true one. During an attack of chlorosis a large number of pale red blood corpuscles are formed, very deticient in iron. These die out only slowly, and it takes weeks, or even months, before they are quite replaced by the newly-formed corpuscles rich in iron. As the pale corpuscles become less and less numerous the hæmoglobin percentage gradually goes up to the normal. In women, however, its normal is only about from 82 to 88 per cent. on Gowers's hæmoglobinometer, and, so far as my experience goes, 100 per cent. hæmoglobin is not very common even in healthy men. Leichtenstein ${ }^{86}$ states that women from 11 to 50 years of age average 8 per cent. less hæmoglobin than men. By the use of iron, therefore, we can scarcely hope to bring the blood of chlorotic women up to 100 per cent. of hæmoglobin, but by a little persistence in treatment we can readily bring it up to between 80 and 90 per cent., which is the average for healthy young women.

These observations were made in Chalmers' Hospital, and my best thanks are due to Dr. George W. Balfour, not only for his kindness in placing beds at my disposal for the treatment of patients, but also for continually affording me the great benefit of his own exceptionally large knowledge and experience of anæmic conditions. To Drs. Basil Orr, Ronald Mackenzie, and Harry Rainy, who, as house-surgeons of the hospital, gave themselves a great deal of trouble on my behalf, I am also much indebted.

\section{A CASE OF PERFORATED GASTRIC ULCER} FOR WHICH GASTRORRHAPHY WAS PERFORMED: DEATH ON THE THIRTYFIRST DAY.

BY HASTINGS GILFORD, F.R.C.S., Reading.

THe following is the report of a case in which a girl, aged 20 , passed successively through the shock of the perforation of a gastric ulcer, followed by abdominal section and gastrorrhaphy,

"Untersuchungen iber den Hæmoglobingehalt des Blutes, Leipzig, 1878.

1 Read before the Reading Pathological society. then through slight creolin poisoning, double parotitis, the operation of gastrotomy, septic pneumonia and diarrhœe, and ultimately died of septicæmia, which supervened upon a final operation for closure of the wound.

At 1.30 1.M. on October 19th, 1892, I was asked to attend a girl who had been seized suddenly with intense abdomina pain. I found her in great agony, with shrunken features, cold and clammy skin, and a small, hard, frequent pulse. The pain was referred to the left epigastric and hypochondriac regions, and was accompanied by tenderness and muscular rigidity. The abdomen was rather contracted than full, and was resonant everywhere. There was nausea, but she had not vomited, though she made occasional gulps like those which precede vomiting, and belched a little wind. The bowels were regular. Respirations were frequent, shallow, and entirely costal. At 9 P.M. she had eaten a hearty supper of bread and cheese, cabbage, and potatoes, and at 12 had been awakened from sleep by the onset of the pain. I elicited the facts that she had vomited on a few occasions about a month before, and that there had then been slight pain in the region of the stomach. I gave her two hypodermic injections of sedative solution of opium, and the pain was soon greatly relieved, but as it disappeared a desire for air, of which she had previously complained became urgent. As the abdomen had now become distended, I had her raised in bed, to her great relief. Two hours afterwards the opiate had to be repeated, and she then vomited half a pint of partially digested food. Meanwhile I made hasty preparations for an early laparotomy, but, finding she was rallying from the shock, I delayed operation until a more convenient season.

At 10.30 A.M. the temperature was $101.6^{\circ}$; the pulse small, hard, 140; the abdomen slightly distended, and tender to pressure in every part. She had not micturated for twelve hours, and there was no hypogastric dulness. The knees were flexed, and ans attempt to straighten them caused pain. Dr. Moody-Ward now saw her, advised laparotomy, and kindly gave chloroform.

An incision was made 3 inches long, 1 inch to the left of, and parallel with, the linea alba, starting half an inch from the margin of the ribs. A quantity of peritoneal fluid gushed out, which on close inspection was found to have oil globules floating on its surface. After flowing a few seconds a few particles of half-digested food began to make their appearance. The blood vessels of the anterior wall of the stomach were very full, the congestion increasing as the lesser curvature was approached. A rough, irregular surface was now felt on the lesser curvature near its middle, and on bringing this into view a quantity of granulation tissue was seen, together with lymph of more recent date, while above it, on the adjacent surface of the liver, were corresponding marks of a previous adhesion. It was thought that the ulcer would be found at this spot, and time was spent in searching for it, but without result. After carefully breaking down some adhesions, and watching the direction of the current which was continuously oozing from the stomach, it became evident that the stream came from a direction nearer the cardiac end. The finger was therefore insinuated among the adhesions of this part, and there the aperture was found. It was situated rather on the posterior than on the anterior aspect, and could not be brought into view at this stage. It was large enough to permit of the introduction of the finger for the exploration of the stomach for other ulcers, but with a negative result. Four silk sutures were passed through the stomach walls half an inch from the edge of the ulcer, and tied 80 as to invert it towards the interior. The abdominal cavity was afterwards irrigated with boiled water, to which, as it was not sufficiently cool, a little creolin (which is said to be an unirritating, non-poisonous antiseptic) was added, the Reading tap water being on that day much too foul to be safely used without some such precaution. A glass drainage tube was then pushed up so that its extremity rested on the site of the sutured ulcer, and the external wound was closed.

For two days after the operation the patient was delirious at times, and had occasional twitchings of the muscles of the face, with upward turning of the eyeballs and spasmodic clenching of the hands. Urine was passed twenty-four hours after the operation, and was of a dark colour, but contained no albumen: specific gravity 1035. The tube was syringed with weak Condy's fluid in boiled water four times a day 
during the first four days. There was at first a large quantity of peritoneal fluid in the dressings, but this discharge soon diminished. On the third day the discharge became offensive, and a piece of stinking lymph was syringed from the tube. Next day the tube was removed, cleansed, and the odour finally got rid of. She was fed by enemata of peptonised milk, of which 6 to 10 ounces were given every four to six hours. On the fifth day 2 ounces of creasote water were given by the mouth in occasional teaspoonful doses, and 3 ounces of plain water on the sixth. On the eighth day the discharge, which had diminished to about 1 drachm at each dressing, began to increase. Water was therefore given by the mouth while the tube was watched, and it was seen that nearly four seconds after swallowing the fluid bubbles of air came through the wound. The water was therefore stopped, but bile-stained mucus exuded from the opening, and was drained off by a siphon drainage tube.

On the tenth day the right parotid began to swell, and the temperature went up to $103.8^{\circ}$; the next day the left gland became inflamed, and she looked exceedingly prostrate and ill. The pulse was 140, small and weak. On the eleventh day she became intolerant of nutrient enemata. Milk given by the mouth flowed in almost undiminished quantity through the wound, and though temporary improvement resulted from feeding through a soft tube passed down the œsophagus beyond the seat of the ulcer, yet the same result ensued after a few feedings. Under these circumstances it was thought advisable to hook the stomach forward into the wound, to pass two silk sutures through its wall, and to form a small opening direct into its cavity. This opening was within 2 inches of the pylorus, and was just large enough to admit a No. 10 catheter, which was pushed on through the pylorus until a sense of resistance was felt-a distance of 10 inches beyond the wound. A drainage tube was left in the upper angle of the wound. Half an ounce of peptonised milk was given through the tube every quarter of an hour, and subsequently 3 ounces of scraped raw beef were added to each pint of milk. On the thirteenth day rectal feeding was resumed during the night, the tube being removed from the pylorus and replaced in the morning. On the fourteenth day crepitation was heard at the left base, fœtid diarrhœa set in, and the temperature became septicæmic. For three days the temperature continued to rise progressively higher in the evenings, and pneumonic signs appeared in the other base.

It was evident that a collection of pus existed in the neighbourhood of the stomach, and that it was advisable to evacuate it if possible. I therefore enlarged the opening in the anterior wall of the stomach and explored that viscus from its inside, but no bulging could be detected at any part within reach of my finger. The manipulation caused so much nausea and unpleasant suffocative feeling that I desisted from a thorough exploration until chloroform had been given. It was now ascertained that the stomach was much misshapen by a transverse constriction near its middle, which rendered it hour-glass in shape. A bulging of the mucous membrane was also felt along the lesser curvature, and a hollow needle was plunged into it in different places, care being taken not to puncture the aorta, which could be felt pulsating behind the stomach. Nothing but blood-stained serum was withdrawn. Next day the patient complained of a nasty taste, and spat up a little pus, and the wound was offensive when it was dressed. I therefore washed out the stomach, and was pleased to find curdy offensive pus in the washings.

The effect of its discharge on the patient was most beneficial, for she immediately felt better-the pneumonia cleared up, the diarrhœa ceased, and the temperature became lower though it was still irregular. She also, for the first time, said she felt hungry. On the twenty-first day the duodenal feedings began to cause nausea. On one occasion the milk had been slightly acidified with hydrochloric acid, but such a free discharge of bile took place through the wound that the acid was abandoned. Another trial was now made, the patient being shifted into various positions on her right side to prevent regurgitation through the pylorus if possible, but always with the same result. It was therefore considered advisable to close the stomach wound and feed first by the rectum and then by the mouth. The small quantity of pus now washed out was no longer offensive, but the temperature still cor- tinued irregular $\left(98^{\circ}\right.$ to $\left.100.8^{\circ}\right)$, indicating that the external wound should remain open. Four silk sutures were passed through the whole thickness of the stomach wall, about half an inch from the margins of the opening, and the external wound was also partially closed, in order to give additional support, strips of plaster being used to secure temporary apposition of the whole of the two surfaces. No pain whatever was felt when the stomach had been incised on the previous occasion, but a few whiffs of chloroform were now given After this operation had been done the patient was seized with a troublesome cough, which threw great strain on the stitches and caused those in the stomach wall to cut through her efforts to restrain the cough making matters worse. She was also showing signs of becoming intolerant of the rectal feeding. Two more deep sutures were put in, so as to secure a good grip of the whole thickness of skin and stomach and mouth feeding was resumed. It was found that by giving 2 ounces of peptonised milk every three hours there was scarcely any leakage through the wound. Rabbit broth and beef tea were then added to milk, and, after two days of this dietary, she was given six oysters a day, and finally three small meals of fish, the solid food being still more satisfactory than theliquid.

This was continued until the sutures began to show signs of tearing through the skin wound, which had not united because of the irritating gastric fluid which oozed up between its surfaces. The temperature was lower and more regular $\left(99.2^{\circ}\right.$ to $\left.100^{\circ}\right)$, and the patient appeared in good condition to withstand a week of rectal feeding. Dr. Moody-Ward, who again saw her at this time, concurred with me in my opinion that the time had come for final closure of the wound. Chloroform was therefore given and the parts explored. All seemed satisfactory, but the walls of the stomach had so. united with the tissues about the wound as to render any separation of them impossible. The edges of the lower two-thirds of the wound had been freshened, and a probe was being used to ascertain the depth of surface at the upper part, when it suddenly ran into a cavity containing about half an ounce of stinking pus. This was found to extend for about $2 \frac{1}{2}$ inches between the stomach and liver, and an attempt was made to clean it out. The probe was used for this purpose, and was employed carefully, while the little finger was passed occasionally into the wound to explore. The cavity was then well syringed with boracic lotion, and a glass drainage tube was passed to its end. The edges of the freshened incision were brought together with five catgut and four silk sutures, passed through the stomach and the whole thickness of the parts respectively. The patient was much collapsed afterwards, and the pupils were widely dilated, but she passed a fair night, and seemed much better in the morning. A sudden change for the worse took place at 10.30 A.M., when she became pale and livid, with sunken eyes and small, feeble, rapid pulse. Stimulants were used, including saline transfusion, but fœtid diarrhœa set in. and she died at 3 P.M. one month from the date of abdominal section, and on her 20 th birthday.

Post-mortem Examination. - No peritonitis was found. The stomach was firmly adherent to the abdominal walls in the immediate neighbourhood of the skin incision, and to the lesser lobe of the liver above. Between the liver and stomach a small abscess was opened, and, adjacent to it, a larger cavitz containing granular offensive material. The liver at this part was soft and light yellow in colour, and its tissue was loaded with pus cells, but no distinct abscesses were found. The stomach was markedly hour-glass in shape, the contraction having been produced by an old ulcer at the middle of the posterior surface. On the greater curvature of the upper compartment of the hour-glass was an ulcer 3 inch in dismeter, and on the lesser curvature, $1 \frac{1}{2}$ inch from the osophagus and more on the posterior aspect than on the anterior, was. the ulcer which had given rise to the necessity for operation. It was a little smaller than the other, and showed no trace of union at any part. The shape of the stomach, together with the situation of this ulcer, explained why the suture yielded and why, when she was first fed by the mouth, almost the whole of the food made its way through the ulcer, for it was so situated as to act as an artificial pylorus to the upper of the two stomachs into which the single cavity had been divided. $A$ third and much smaller ulcer was found near the middle of the lesser curvature. 
Remarks. - I think the partial success of this case was mainly due to the fact that the girl was lying in bed when rupture took place, and that she remained on her back until a ter the distension of the intestines had shut off most of the extravasated matter from the lower peritoneal cavity. Some food undoubtedly escaped into it, and there was evidence of mild peritonitis there, both before and after the operation. As bearing on this point, I will mention a case which came under my observation, in which a woman was walking when rupture of a gastric ulcer took place, and great difficulty in diagnosis arose from the fact that, notwithstanding my leading questions, she persisted in her statements that the seat of first pain and of greatest pain and tenderness was in the left iliac region. There was no history of gastric trouble beyond slight dyspepsia of an indefinite character, and there was the misleading positive history of a lump situated in the left iliac region, which had been noticed to "gradually increase in size until it reached that of an orange." I had made preparations to cut down upon this part with vague ideas of finding a ruptured abscess or ovarian cyst (though the possibility of its being due to ruptured gastric ulcer was still entertained), when the patient got beyond all help and died rapidly. Indeed, Mr. Truman, who kindly acted on my responsibility, was actually giving chloroform just before death took place. She was then fully conscious, and her pulse was 150 . I thought the anæsthetic might diminish the effect of the shock, but it had no such result. I am glad, however, to see that my action in this case has its precedent, for Mr. Taylor of Birmingham ${ }^{1}$ operated with success upon a patient who was apparently sinking, the lips being livid, the extremities cold, and the pulse uncountable. After death $I$ found an ulcer on the lesser curvature, - Sout the site of intensest peritonitis was in the left iliac fossa and it was evident that as the woman was upright when rupture had taken place, the contents of the stomach had fallen to the lower part of the abdomen.

I could not account for the parovaritis, for it was not sup purative, it was bilateral, and there were no other signs of septic poisoning, except perhaps the temperature, which remained over $100^{\circ}$. But Dr. Moody-Ward referred me to an interesting vaper by Mr. Stephen Paget, ${ }^{2}$ in which he shows that parovaritis is a rare complication of pelvic and abdominal disease, and may occur independently of septic causes. Mr. Taylor's is the first successful case recorded of operative treatment of ruptured gastric ulcer. The incision was from the umbilicus to the pubes, and the stomach was left alone. The patient survived, not only the operation, but septic pneumonia, subdiaphragmatic and pleural abseesses, and septic intoxication from insanitary surroundings, and died ultimately of intestinal obstruction, the result of the rupture, just two months afterwards. Mr. Bartlett, ${ }^{3}$ of Birmingham, operated upon a case of Dr. Saundby's, but without success, but a successful case has recently been recorded by Mr. Gilbert Barling. ${ }^{4}$ Dr. Sinclair's $s^{5}$ case died on the sixth day after an operation by enedian incision below the umbilicus. Dr. Dreschfeld ${ }^{6} \mathrm{re}^{-}$ commends a preliminary puncture with a Southey's trocar, as * means of diagnosis, to ascertain by its odour if the extravasated gas comes from the stomach or intestines. He points to the fact that vomiting is almost invariably absent in this form of perforation, and mentions a case of Czerny's $\mathrm{s}^{\mathrm{T}}$ in which the operation was performed by median epigastric incision five days after perforation, and death resulted from fresh peritonitis in four days. Mikulicz ${ }^{8}$ reports an extraordinary case in which gas, smelling strongly of alcohol, escaped through a cannula which was pushed into the abdominal cavity for diagnosis, and the abdomen was opened first from the navel to the symphysis, and the incision was afterwards extended to the xiphoid cartilage. A clean rupture was then found along the lesser curvature, apparently the result of the bursting of an overdistended stomach, without previous ulceration. Death took place in three hours. Billroth ${ }^{9}$ performed gastrorrhaphy for gunshot wound of the stomach, but death ensued in twenty-

$$
\begin{aligned}
& \text { BRITISH MEDICAL JOURNAL, 1886, voI. i, p. } 183 \\
& 2 \text { Birmingham Medical Review, 1888, p. } 155 \\
& 3 \text { Birmingham Medical Keview, 1888, p. } 183 . \\
& \text { BRITISH MEDICAL JOURNAL, ii, 1892, p. } 1011 \\
& \text { - Med. Chron, 1889, May. } \\
& 7 \text { Berlin. Klin. W och. } \\
& 8 \text { Volkmann's Sam. klin. Vort., p. 2,310, } 1885 . \\
& \text { Magenoperationen, } 18808 \mathbf{8}
\end{aligned}
$$

eight hours. Dr. Parsons ${ }^{10}$ stitched the margins of a ruptured ulcer to his abdominal incision. Death resulted in six days. He mentions two cases of traumatic perforation of the stomach in the practices of Professor Kocher and Dr. Ball respectively. in the former of which recovery took place.

\section{THE DIRECT PYRAMIDAL TRACTS OF THE SPINAL CORD.}

BY R. T. WILLIA MsON, M.D.Lond., M.R.C.P., Medical Registrar, Manchester Royal Infirmary.

The British Medicax Journat of April 5th, 1890 , contains a leading article on the functions of the above tracts, and also the notes of a most interesting case, recorded by Dr. Macewen, which he regards as giving some support to the theory that the function of the direct pyramidal tract is to convey motor fibres to the arms and probably to the intercostal muscles.

In Professor Macewen's case " the intrinsic muscles of the forearm, wrist, and hand were absolutely paralysed." There was paresis of the supra- and infraspinati, deltoids, biceps, brachiales antici, and supinators. The legs were not affected. Macewen localises the lesion in the cord at the region of the seventh and eighth cervical and first dorsal, and suggests that the lesion was one implicating the anterior (or direct) pyramidal tracts at this region, and that the crossed or lateral pyramidal tracts were unaffected. He thinks the symptoms may be explained by accepting the theory that the direct pyramidal tracts are chiefly related to the innervation of the arms.

The direct or anterior pyramidal tracts (columns of Türck) consist of fibres which have not decussated at the medulla; the fibres, which have decussated passing down the crossed pyramidal tracts. The direct pyramidal tracts gradually diminish from above downwards, and as a rule cease about the middle dorsal region, but sometimes pass down to the lower dorsal region. Hence, as pointed out by Tooth, this tract must have some other function in addition to the innervation of the arms. Macewen points out "that the direct tracts generally continue down the cord to the middle of the dorsal region, where the anterior tracts would terminate did they supply the principal respiratory intercostals." He suggests that in addition to the innervation of the arms they may also innervate the intercostals.

Some time ago, through the kindness of the late Dr. Ross, I had the opportunity of observing clinically and of making the pathological examination in a case of spinal cord disease, in which the direct pyramidal tracts were markedly degeneration at the part at which Macewen placed the lesion in his case, and yet the arms were not paralysed.

J. T., aged 39, was admitted under the care of Dr. Ross at the Manchester Royal Infirmary, July 19th, 1890.

Previous History.-About two years before admission the patient first noticed slight numbness in the fingers of both hands, then the legs be came weak; they gradually became worse, and five or six months before admission he was compelled to give up wcrk on aocount of loss of power in his legs. There was no history of cramps in the legs, pains in the back no history of alcoholic excess, syphilis or diphtheria.

State on Admission. - There was a little odema in both legs. The patient was quite unable to stand. He was unable to raise the legs in. the extended position from the bed. He was able to flex and extend the legs at the hips and knees very slightly. There was only the slightest movement when he attempted to dorsiflex the feet. The knee-jerks were absent, and there was no ankle clonus. The plantar reflexes were feeble. There was no rigidity of the legs, and no pain in the legs except when they were moved. He was able to teel and localise the touch with a pin's head (if the pressure were moderate), and was able to distinguish between the the pressure were moderate, and a d ablo head was often not reco pirised. The patient was able to perform al the head was often not recognised. The patient was able to perform all the movements of the arms with the exception of abduction at the right shoulder. This he was unable to perform on account of pain in the right shoulder.joint. He was able to feel and localise the touch of a pin's head on bath hands and arms. The diameter of the pupil on the right side was glightly greater than on the leit; ; both reacted quite well to light. abdomen, and urine.

At a later date there was incontinence of urine, and this continued until his death. Cystitis occurred, and a small bedsore formed over the sacrum. The paralysis of the legs persisted, and slight anæsthesia occurred in the legs, but there was no paralysis of the arms. The kneejerks were absert up to the last. There was no ankle olonus, and the plantar reflexes were absent. The patient became delirious, and passed into a semi-conscious condition. Death occurred on October 12 th.

\footnotetext{
${ }^{10}$ Dub. MId. Journ., vol. 1i, p. 26, 1892.
} 